\title{
Comparison between different thickness umbrella-shaped expandable radiofrequency electrodes (SuperSlim and CoAccess): Experimental and clinical study
}

\author{
MASAHIKO KODA, SHIHO TOKUNAGA, TOMOMITSU MATONO, TAKAAKI SUGIHARA, \\ TAKAKAZU NAGAHARA and YOSHIKAZU MURAWAKI
}

Second Department of Internal Medicine, School of Medicine, Tottori University, Yonago 683-8504, Japan

Received July 13, 2011; Accepted August 24, 2011

DOI: $10.3892 / \mathrm{etm} .2011 .347$

\begin{abstract}
The purpose of the present study was to compare the size and configuration of the ablation zones created by SuperSlim and CoAccess electrodes, using various ablation algorithms in ex vivo bovine liver and in clinical cases. In the experimental study, we ablated explanted bovine liver using 2 types of electrodes and 4 ablation algorithms (combinations of incremental power supply, stepwise expansion and additional low-power ablation) and evaluated the ablation area and time. In the clinical study, we compared the ablation volume and the shape of the ablation zone between both electrodes in 23 hepatocellular carcinoma (HCC) cases with the best algorithm (incremental power supply, stepwise expansion and additional low-power ablation) as derived from the experimental study. In the experimental study, the ablation area and time by the CoAccess electrode were significantly greater compared to those by the SuperSlim electrode for the single-step (algorithm $1, p=0.0209$ and 0.0325 , respectively) and stepwise expansion algorithms (algorithm 2, p=0.0002 and <0.0001, respectively; algorithm 3, p=0.006 and 0.0407, respectively). However, differences were not significant for the additional low-power ablation algorithm. In the clinical study, the ablation volume and time in the CoAccess group were significantly larger and longer, respectively, compared to those in the SuperSlim group ( $\mathrm{p}=0.0242$ and 0.009 , respectively). Round ablation zones were acquired in $91.7 \%$ of the CoAccess group, while irregular ablation zones were obtained in $45.5 \%$ of the SuperSlim group $(p=0.0428)$. In conclusion, the CoAccess electrode achieves larger and more uniform ablation zones compared with the
\end{abstract}

Correspondence to: Dr Masahiko Koda, Second Department of Internal Medicine, School of Medicine, Tottori University, 36-1 Nishicho, Yonago 683-8504, Japan

E-mail: masakoda@grape.med.tottori-u.ac.jp

Key words: CoAccess electrode, SuperSlim electrode, radiofrequency ablation, hepatocellular carcinoma, ablation zone, umbrella-shaped expandable electrode
SuperSlim electrode, though it requires longer ablation times in experimental and clinical studies.

\section{Introduction}

Radiofrequency (RF) ablation (RFA) is recognized as a minimally invasive treatment for hepatocellular carcinoma (HCC) (1-5). The ideal goal of RFA for HCC is to obtain a reproducible ablation volume that encompasses the tumor, surrounded by a margin of hepatic parenchyma $(6,7)$. The critical steps in attaining this goal are correct electrode positioning according to the planned ablation algorithm and the acquisition of a constant ablation volume. In RFA, the physician positions the electrode needle in the center of the tumor under ultrasound guidance. However, insufficient ablation may occur when the ablation volume is more irregular or smaller than expected.

One of several commercially available RF devices is the RF 3000TM RFA system (Boston Scientific Corporation, Natick, MA, USA) using an umbrella-shaped expandable electrode (8). The conventional electrode (LeVeen eletrode) has a $15 \mathrm{G}$ cannula and 10 tines and was the first to become commercially available, but it is thicker and less sharp than another RFA device (internally cooled electrode, Radionics, Burlington, MA, USA). Two new umbrella-shaped expandable electrodes have become available as improved versions; one of these, the SuperSlim electrode, has a thinner cannula and thinner tines compared to the conventional electrode and became available in Japan in October 2003. It enables easy puncture into the tumor and may reduce severe complications, such as hemorrhaging (9). The other, the CoAccess electrode, is used in combination with a coaxial insulated needle, though it has the same thick cannula and tines as the conventional LeVeen electrode. It became available in Japan in August 2005. The 14-gauge coaxial insulated needle enables easy puncture, and is highly cuspidate due to its diamond-cut tip, even though its coaxial needle is 3 gauges thicker compared to that of the SuperSlim electrode. Although both electrodes offer improved puncture compared with the conventional LeVeen electrode, no previous studies have investigated the ablation effect of the two electrodes. A number of ablation algorithms have been proposed to achieve more efficient ablation using these electrodes; e.g., 
the multi-step expansion method or additional low-power ablation $(10,11)$. The purpose of the present study was to investigate the size and configuration of the ablation zones created by the SuperSlim and CoAccess electrodes, using various ablation algorithms in ex vivo bovine liver and in clinical cases.

\section{Material and methods}

Experimental study. We compared two types of improved LeVeen ${ }^{\circledR}$ electrode (Boston Scientific Corporation, Natick, MA, USA) incorporating a 3-cm array: the SuperSlim and the CoAccess electrode (Fig. 1). The SuperSlim electrode has a $17 \mathrm{G}$ cannula and 10 electrode tines (tine diameter: proximal site, $0.305 \mathrm{~mm}$; distal site, $0.104 \mathrm{~mm}$ ), while the CoAccess electrode has a $15 \mathrm{G}$ cannula and 10 electrode tines (tine diameter: proximal site, $0.34 \mathrm{~mm}$; distal site, $0.162 \mathrm{~mm}$ ) similar to the conventional LeVeen electrode. In the CoAccess electrode procedure, the liver was punctured initially with a $14 \mathrm{G}$ coaxial insulated needle, the stylet was removed, and the CoAccess electrode was inserted through the coaxial needle. The cannula and the electrode tines of the SuperSlim electrode are thinner compared to those of the CoAccess electrode.

Explanted fresh bovine livers were prepared for ablation studies: $2 \mathrm{~kg}$ of liver were placed on a copper plate with 2 grounding pads at room temperature (Fig. 2). Under sonographic guidance, the electrode was inserted into the bovine liver from the upper side. We compared the ablative states between the SuperSlim and the CoAccess electrodes, both with a $3-\mathrm{cm}$ array diameter, and the following 4 algorithms. These 4 algorithms were selected based upon manufacturerrecommended and existing clinical algorithms, namely the combination of incremental power supply, stepwise expansion and additional low-power ablation: algorithm 1, the tines were fully expanded and RF energy was then applied to the tissue using an initial power setting of $20 \mathrm{~W}$, which was subsequently increased in increments of $10 \mathrm{~W} / \mathrm{min}$ until the impedance rose markedly; algorithm 2, RF energy was applied to the tissue using a fixed power setting of $20 \mathrm{~W}$. The electrode tines were expanded incrementally in 3 steps: array diameter was $15 \mathrm{~mm}$ at the first step, $25 \mathrm{~mm}$ at the second, and $30 \mathrm{~mm}$ (fully expanded) at the third. At each step, RF energy was applied at $20 \mathrm{~W}$ until the impedance rose markedly; algorithm 3, the tines were expanded incrementally as in algorithm 2. RF energy was then applied to the liver using an initial power setting of $20 \mathrm{~W}$, which was subsequently increased in increments of $10 \mathrm{~W} / \mathrm{min}$ until the impedance rose markedly, at each step; algorithm 4, ablation additional to that performed in algorithm 3 was applied at $70 \%$ of maximum power at the full extension of the array until the impedance rose markedly, or for $15 \mathrm{~min}$.

During ablation, RF energy and ablation time were recorded. After ablation, the liver was cut along the puncture line of the RF electrode. To determine the area ablated by RFA, the ablation area was measured from an image of the cut surface analyzed using the freely available Image J software (National Institute of Health, Bethesda, Maryland, USA) (http://rsbweb.nih.gov/ij/index.html). This experimental study was performed by a single operator (M.K.).

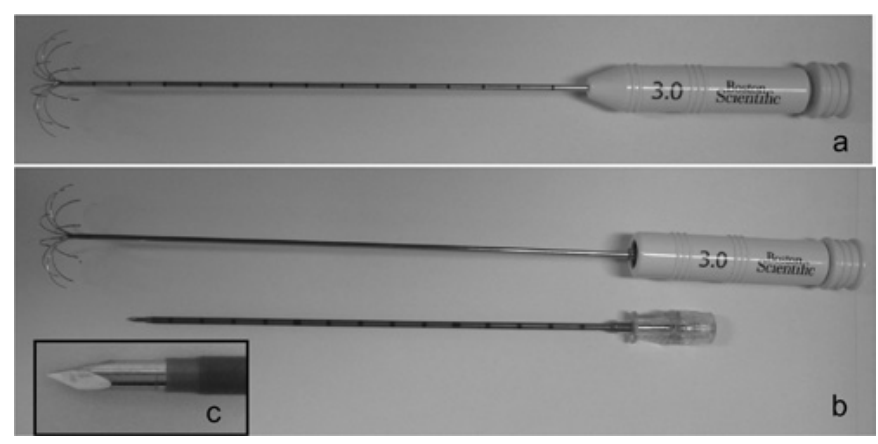

Figure 1. Image of the radiofrequency (RF) electrodes used in the present study (LeVeen Electrode System). (a) Array $(3 \mathrm{~cm}$ ) SuperSlim electrode. (b) The array $(3 \mathrm{~cm})$ CoAccess electrode system consists of a CoAccess electrode (top) and coaxial insulated needle (bottom). (c) Magnified view of the diamond-cut tip of the coaxial insulated needle.

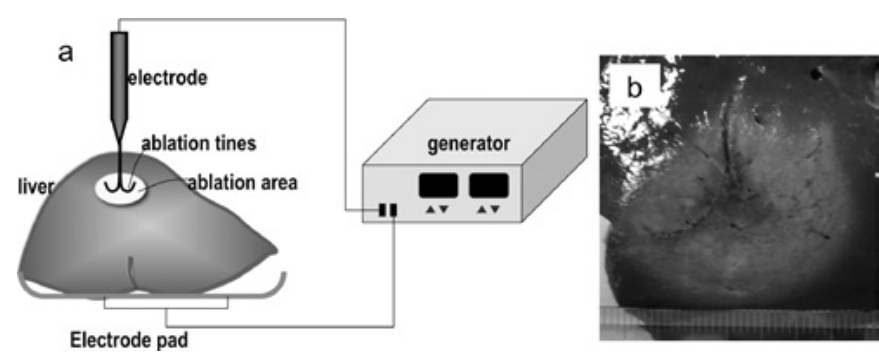

Figure 2. (a) Diagram showing the system for ablating ex vivo bovine liver. A block of bovine liver is placed on a copper plate with grounding pads, and the electrode needle is positioned in the liver from the upper side under sonographic guidance. We measured the ablation time and area for each algorithm. (b) Cut surface of the ablated liver, cut along the puncture line of the radiofrequency $(\mathrm{RF})$ electrode. The ablation area was measured using Image J software.

Clinical study. From January 2004 to November 2006, 243 nodules in 186 patients were treated by RFA. Patients included in this study fulfilled the following criteria: i) the size of the HCC nodule was $<3 \mathrm{~cm}$ in diameter, ii) the number of HCC nodules was $\leq 3$, iii) no portal thrombosis or extrahepatic metastasis were present, and iv) RFA was performed with 3-cm array SuperSlim or CoAccess electrodes at one position, that is, overlapping ablation at another position was not done to enlarge ablation volume. The SuperSlim group consisted of patients treated from January 2004 to August 2005 and the CoAccess group of patients treated from September 2005 to November 2006. A total amount of 24 patients (12 patients in the SuperSlim group and 12 in the CoAccess group, respectively) were consecutively enrolled to this study; 1 patient in the SuperSlim group who was not evaluated by enhanced CT after treatment was excluded due to contrast agent allergy. The study was approved by the Ethics Committee of our institute (no. 1607) and this analysis was conducted retrospectively. The nature of the study was fully explained to the patients, and informed consent was obtained. We analyzed 23 patients with HCC who were treated with percutaneous RFA at our hospital. The preoperative clinical features of these 23 patients are listed in Table I. All patients had underlying chronic liver disease: chronic hepatitis in 4 patients and cirrhosis in 19, Child-Pugh grade A in 14 patients and grade B in 5. Hepatitis $B$ surface antigen was positive in 4 patients, hepatitis $C$ virus 
Table I. Characteristics of patients in the SuperSlim and CoAccess groups.

\begin{tabular}{|c|c|c|c|}
\hline Characteristics & $\begin{array}{l}\text { SuperSlim } \\
\qquad(\mathrm{n}=11)\end{array}$ & $\begin{array}{l}\text { CoAccess } \\
(n=12)\end{array}$ & p-value \\
\hline Male/female & $9 / 2$ & $10 / 2$ & NS \\
\hline Age (years) & $67.7 \pm 8.70$ & $73.2 \pm 7.80$ & NS \\
\hline \multicolumn{4}{|l|}{ Etiology } \\
\hline Hepatitis B & 3 & 1 & \multirow{4}{*}{ NS } \\
\hline Hepatitis C & 6 & 11 & \\
\hline Alcoholic & 1 & 0 & \\
\hline Cryptogenic & 1 & 0 & \\
\hline \multicolumn{4}{|l|}{ Underlying liver disease } \\
\hline Chronic hepatitis & 0 & 4 & \multirow{4}{*}{ NS } \\
\hline Cirrhosis & 11 & 8 & \\
\hline Child-Pugh A & 9 & 5 & \\
\hline Child-Pugh B & 2 & 3 & \\
\hline Tumor size (mm) & $18.5 \pm 5.90$ & $21.3 \pm 3.60$ & NS \\
\hline Mean follow-up period (months) & $38.5 \pm 10.7$ & $32.1 \pm 8.95$ & NS \\
\hline
\end{tabular}

Data are expressed as the means \pm standard deviation (SD); NS, not significant.

antibody was positive in 17 , and the remaining 2 patients were cryptogenic and alcoholics, respectively. There were no significant differences in tumor size between the 2 groups.

Prior to RFA, all patients underwent US, enhanced CT and MRI, and/or CT scan under arteriography and portography through the superior mesenteric artery via a catheter. On enhanced CT or MRI, hyper-enhancement in the arterial phase with washout in the portal phase was designated as HCC.

RFA algorithm. After local anesthesia, RFA therapy was performed under sonographic guidance using a real-time convex scanner with $3.75-\mathrm{MHz}$ probes (SSA-360A; Toshiba, Tokyo, Japan) and a biopsy guide device. We used the RF3000 generator system with 2 types of electrodes (CoAccess and SuperSlim) according to algorithm 4, which was the most efficient algorithm from the results of the experimental studies.

In brief, the electrode was positioned in the tumor and the array was then expanded in 3 steps of array diameters (15, 25 and $30 \mathrm{~mm}$ ). In the first step, hooks were deployed at an array diameter of $15 \mathrm{~mm}$ and RF power was initially applied at $30 \mathrm{~W}$, which was increased by $10 \mathrm{~W} / \mathrm{min}$ until the impedance rose markedly. The second step began at the RF power level reached in the first step, and RF power was increased by $10 \mathrm{~W} / \mathrm{min}$ until the impedance rose markedly. This cycle was repeated at each step to full extension of the array. Additional ablation was applied at $70 \%$ of maximum power until the impedance rose markedly or for $15 \mathrm{~min}$.

Imaging analysis. For post-treatment evaluation, helical multiphasic CT examinations were performed 1 month after RFA using a multi-detector scanner (Somatom Plus; Siemens, Forchheim, Germany) with the following imaging protocol: tube voltage, $120 \mathrm{kV}$; tube current, automatic $\mathrm{mA}$ setting; reconstruction section and interval thickness, $3 \mathrm{~mm}$; detector configuration, 32x1 mm; pitch, 27; and gantry speed,
$0.5 \mathrm{sec}$ per rotation. Unenhanced CT images were acquired, followed by triple-phase contrast-enhanced images during power injection of $100 \mathrm{ml}$ of iopamidol (Iopamiron; NihonSchering, Osaka, Japan) at a rate of $2.7 \mathrm{ml} / \mathrm{sec}$. The entire liver was scanned 3 times. Early arterial phase imaging was initiated at $10 \mathrm{sec}$, late arterial phase imaging at $20 \mathrm{sec}$, and portal venous phase imaging at $120 \mathrm{sec}$ after initiation of the injection. All scans were obtained with a $5 \mathrm{~mm}$ slice pitch. After treatment, the volume of the RF-induced ablation zone was evaluated using Image $\mathrm{J}$ by measuring the unenhanced area for each slice of dynamic CT and summing these values. The shape of the RF-induced ablation zone was classified into 2 types: irregular and round. We defined 'round' as a smooth spherical contour in the margin of the ablation zone (Fig. 3a) and 'irregular' as a lobulated, non-spherical contour (Fig. 3b). Post-ablational CT studies were independently reviewed on a compute workstation by two abdominal imaging radiologists (M.K. and S.T., who had 25 and 11 years experience, respectively). The reviewers knew the diagnosis of HCC but were blinded to other clinical data. Discrepancies between the two readers were resolved by discussion to reach consensus.

Patients were followed-up every 3 months with measurement of serum $\alpha$-fetoprotein (normal: $<12 \mathrm{ng} / \mathrm{ml}$ ) and des- $\gamma$-carboxy prothrombin (normal: $<40 \mathrm{mAU} / \mathrm{ml}$ ) levels, and enhanced CT or enhanced MRI. When recurrence was suspected, the diagnosis of intrahepatic recurrence was made in the case of positive findings in at least two of the following: CT, MRI, sonography, angiography and needle biopsy.

Statistical analysis. All measurements in the experimental studies were performed 5 times except for algorithm 2 in the CoAccess electrode, where one measurement was excluded due to the failure of ablation. The results are shown as the means \pm standard deviation (SD). Statistical comparisons for ablation time and the area ablated by RFA were made using the 
Table II. Total ablation time (sec) and area $\left(\mathrm{mm}^{2}\right)$ by SuperSlim and CoAccess electrodes in ex vivo bovine liver for the 4 algorithms.

\begin{tabular}{|c|c|c|c|c|c|c|}
\hline Algorithms & & SuperSlim & No. & CoAccess & No. & $\mathrm{p}$-value \\
\hline \multirow[t]{3}{*}{1} & Total ablation time & $301 \pm 99$ & \multirow{3}{*}{5} & $518 \pm 120$ & \multirow{3}{*}{5} & 0.0325 \\
\hline & Maximal RF power (w) & $57.5 \pm 15.0$ & & $83.7 \pm 11.1$ & & 0.0306 \\
\hline & Total ablation area & $647 \pm 340$ & & $1062 \pm 149$ & & 0.0209 \\
\hline \multirow[t]{2}{*}{2} & Total ablation time & $532 \pm 118$ & \multirow[t]{2}{*}{5} & $1326 \pm 189$ & \multirow[t]{2}{*}{4} & $<0.0001$ \\
\hline & Total ablation area & $512 \pm 111$ & & $1037 \pm 142$ & & 0.0002 \\
\hline \multirow[t]{2}{*}{3} & Total ablation time & $280 \pm 51$ & \multirow[t]{2}{*}{5} & $447 \pm 117$ & \multirow[t]{2}{*}{5} & 0.0407 \\
\hline & Total ablation area & $515 \pm 59$ & & $723 \pm 22$ & & 0.0006 \\
\hline \multirow[t]{2}{*}{4} & Total ablation time & $406 \pm 51$ & \multirow[t]{2}{*}{5} & $423 \pm 89$ & \multirow[t]{2}{*}{5} & 0.7260 \\
\hline & Total ablation area & $587 \pm 198$ & & $756 \pm 66$ & & 0.1080 \\
\hline
\end{tabular}

Mann-Whitney U-test with Statview software (SAS Institute, Cary, NC, USA).

\section{Results}

Experimental study. The intra-observer coefficient of variation for the ablation area and time were 10.7 and $9.8 \%$ in SuperSlim electrodes, and 4.7 and $8.5 \%$ in CoAccess electrodes, respectively. The results of ablation by SuperSlim and CoAccess electrodes for each algorithm are shown in Table II. The total ablation time and area of the ablation zone were significantly greater for the CoAccess electrode compared to the SuperSlim electrode for algorithms 1-3 (Table II; $p<0.05$ for all comparisons). In algorithm 4, there was no difference in the total ablation time between the CoAccess and SuperSlim electrodes $(\mathrm{p}=0.726)$. The ablation area by the CoAccess electrode was larger compared to that by the SuperSlim electrode, although this difference was not statistically significant $(\mathrm{p}=0.108)$.

Clinical study. We performed retrospective evaluation of RFA with the CoAccess and the SuperSlim electrodes. In all patients in both groups, the increased impedance was achieved at each step of ablation. Mean ablation volumes in the CoAccess group were significantly larger compared to those in the SuperSlim group (CoAccess, $22.6 \pm 9.1 \mathrm{~cm}^{3}$; SuperSlim, $14.8 \pm 5.9 \mathrm{~cm}^{3}$; $\mathrm{p}=0.0242)$. Ablation times in the CoAccess group were significantly longer compared to those in the SuperSlim group (CoAccess, 21.1 $\pm 4.1 \mathrm{~min}$; SuperSlim, 15.7 $\pm 4.8 \mathrm{~min} ; \mathrm{p}=0.009$ ).

Dynamic CT after 1 month showed no residual tumor in all HCCs treated with RFA in both groups. In the CoAccess group, the shape of the ablation zone was round in 11 (91.7\%) of 12 nodules and irregular in the remaining nodule (9.3\%). In the SuperSlim group, the shape of the ablation zone was round in $6(54.5 \%)$ of 11 nodules and irregular in $5(45.5 \%)$. There was significant difference between the 2 groups regarding the shape of the ablation zone ( $\mathrm{p}=0.0428$ ) (Fig. 3).

On enhanced CT 14 months after RFA, local tumor progression was detected in only 1 of the 11 nodules treated using a SuperSlim electrode. There was no local tumor progression in any nodule treated using a CoAccess electrode. There was no significant difference. There were no severe complications in either group. Five patients (45\%) in the SuperSlim group and

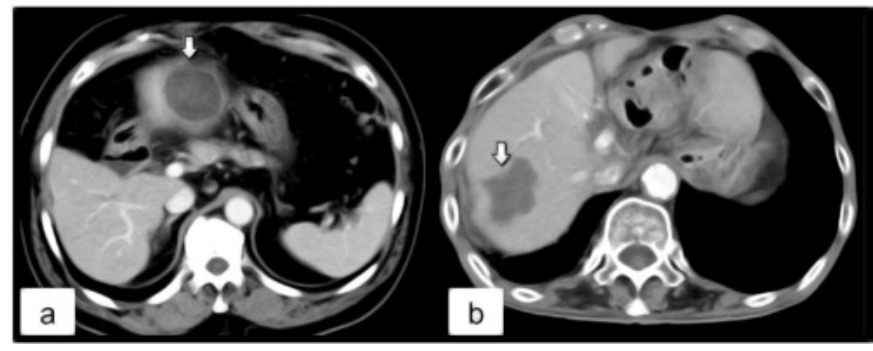

Figure 3. CT images of representative clinical cases treated by CoAccess and by SuperSlim electrodes. (a) Female (75 years old) with a $2.2-\mathrm{cm}$ hepatocellular carcinoma (HCC) in liver segment II, treated using a $3-\mathrm{cm}$ CoAccess electrode. Arterial phase of dynamic $\mathrm{CT}$ reveals a round-shaped ablation zone (arrow). (b) Male (76 years old) with a 2-cm HCC in liver segment VII, treated using a 3-cm SuperSlim electrode. Arterial phase of dynamic CT reveals the irregular outline of the ablation zone (arrow).

7 patients (58\%) in the CoAccess group had low or moderate grade fever.

\section{Discussion}

In RFA, several needle electrodes have been developed, i.e., multitined expandable or cooled single electrodes in an attempt to increase the area of tissue destruction obtained with one RF delivery (8). Furthermore, optimization of the algorithm to achieve appropriate balance among ablation size, duration and precision is a requirement for developing rational strategies for tumor ablation (12-14). For the expandable electrode system, we must take into account several parameters, including the defined increments of tine extension, the duration of RF application at each tine extension and the increment of RF supply. The conventional and manufacturer-recommended algorithm for expandable electrode is the combination of stepwise expansion, incremental RF supply and additional low-power ablation.

The findings of the present study show that RF electrode and parameter selections may influence the ablation area and time. In the clinical setting, we aimed to obtain a large ablation area to achieve complete tumor necrosis, while decreasing the ablation time to reduce the patient's pain during ablation. In the experimental study, we compared the SuperSlim and CoAccess electrodes with regard to the ablation area and time for the 
application of RF energy to the liver, using several treatment algorithms. In algorithms 1 and 2, the CoAccess electrode required significantly longer ablation time compared to the SuperSlim electrode. By contrast, ablation areas achieved using the CoAccess electrode were significantly larger compared to those using the SuperSlim electrode. These results suggest that prolonged ablation time leads to enlargement of the ablation area, which is reported to change with the duration of treatment and probe gauge (12). With increased time, there is additional diffusion of heat and greater tissue ablation until maximum heat diffusion is achieved. Furthermore, there is a linear correlation between the needle gauge and the ablation area (13). The expandable tines of the SuperSlim electrode are thinner compared to those of the CoAccess electrode, and the tissue impedance by the SuperSlim electrode rose earlier compared to that by the CoAccess electrode. Consequently, ablation areas achieved by SuperSlim electrodes are smaller compared to those by CoAccess electrodes.

Algorithm 3 employs the stepwise extension technique proposed by Kobayashi et al (10) to obtain more efficient ablation in a shorter period. Using this technique, a marked increase in tissue impedance is always achieved at each step of extension, and the total time required for ablation is less than the ablation time in the single-step method, although we found no significant difference between the two methods regarding ablation area. Berber et al (15) reported that for the ablation of tumors larger than $3 \mathrm{~cm}$ in diameter, ablation using an initial smaller deployment of $20 \mathrm{~mm}$ to create a nucleus of ablation can result in a larger ablation area in a shorter total ablation time, compared with an initial larger deployment of $30 \mathrm{~mm}$ with slower advancement to the final diameter. Kotoh et al (16) reported that multi-step ablation requires a shorter time compared to single-step ablation. Based on the findings of these studies, we used a stepwise extension technique in the clinical setting. Using algorithm 3, the ablation time and area by the SuperSlim electrode were shorter and smaller, respectively, than those by the CoAccess electrode.

In algorithm 4, in which additional ablation was applied at $70 \%$ of maximal power, ablation areas by the SuperSlim electrode were enlarged (although not significantly) relative to those by the CoAccess electrode, although ablation times for the SuperSlim electrode were longer for algorithm 4 than for 3. According to these experimental results, we applied algorithm 4 to the clinical RFA treatment.

In the clinical study, ablation volumes in the CoAccess group were significantly larger compared to those in the SuperSlim group, although no significant difference in the ablation area was found using the same ablation algorithm in the experimental study. One possible explanation for this discrepancy is that hepatic blood flow may have a cooling effect and could thus have influenced the ablative state in the clinical cases. By contrast, ex vivo bovine liver was not perfused with blood flow (17-19). Previous studies have demonstrated that a reduction in blood supply, such as in transcatheter arterial chemoembolization, results in enlarged ablative volume (20-22). The cooling effect of the blood perfusion in the clinical cases may have disclosed the advantage of the CoAccess electrode masked in the ex vivo experiments. The SuperSlim electrode may not be able to provide sufficient energy deposition to achieve appropriate tissue heating. Solazzo et al (13) showed that the thickness of the electrode limits energy deposition even if a high-output generator is used.

The shape of the ablation zone is important in achieving complete necrosis of HCC. In RFA with an expandable electrode, the edge of the ablation zone is initially concave between the tines as ablation begins at each expanded tine before encompassing the lesion between the tines, becoming convex after sufficient ablation. In the present clinical study, most of the ablation zones by the CoAccess electrode were round in shape. By contrast, $45 \%$ of the ablation zones by the SuperSlim electrode were irregular, even for algorithm 4 . As the expanded tines of a conventional LeVeen electrode are the same as those of the CoAccess electrode, our results for the CoAccess electrode are in agreement with those of Kobayashi et al, who reported that all ablation zones by a conventional LeVeen electrode are sphere-shaped (10). As the tissue impedance by the SuperSlim electrode rose early, sufficient outward enlargement of the ablation zone could not be achieved. The other possibility is that the complete hook deployment is interrupted by tumor capsule or fibrotic tissue in the liver due to thinner tines. It may be possible to obtain a more uniform ablation zone by performing overlapping ablation with the SuperSlim electrode; i.e., after ablation at full extension, the expanded tines are closed, rotated and redeployed at full extension, after which RFA is performed once again.

The present study has certain limitations. Firstly, the results of the ex vivo liver ablation may lead to overestimations of the achievable results in clinical practice due to the lack of blood flow (cooling effect). Secondly, due to the fact that we could not measure the ablation zone in all three dimensions in the experimental study, it was difficult to assess the true volume and ultimate shape of the ablation zones. Thirdly, the in vivo experimental study, which is more similar to clinical practice, could not be performed. Fourthly, our clinical study involved a small number of patients. We could not ethically continue to compare both electrodes due to statistical differences in the ablative states between both electrodes.

In conclusion, by comparing the ablative states achieved by the SuperSlim and CoAccess electrodes in ex vivo bovine liver and clinical cases, we demonstrated that ablation zones achieved by the CoAccess electrode were larger and more uniform in shape compared to those achieved by the SuperSlim electrode, though they required a longer ablation time. We consider that the CoAccess electrode is more useful compared to the SuperSlim electrode for acquiring complete tumor necrosis.

\section{References}

1. Vivarelli M, Guglielmi A, Ruzzenente A, et al: Surgical resection versus percutaneous radiofrequency ablation in the treatment of hepatocellular carcinoma on cirrhotic liver. Ann Surg 240: 102-107, 2004.

2. Curley SA, Izzo F, Ellis LM, Vauthey JN and Vallone P: Radiofrequency ablation of hepatocellular cancer in 110 patients with cirrhosis. Ann Surg 232: 381-391, 2000.

3. Gervais DA, Goldberg SN, Brown DB, Soulen MC, Millward SF and Rajan DK: Society of interventional radiology position statement on percutaneous radiofrequency ablation for the treatment of liver tumors. J Vasc Interv Radiol 20: 3-8, 2000.

4. Livraghi T, Meloni F, Di Stasi M, et al: Sustained complete response and complications rates after radiofrequency ablation of very early hepatocellular carcinoma in cirrhosis: is resection still the treatment of choice? Hepatology 47: 82-89, 2008. 
5. Bhardwaj N, Strickland AD, Ahmad F, Dennison AR and Lloyd DM: Liver ablation techniques: a review. Surg Endosc 24: 254-265, 2010

6. Nakazawa T, Kokubu S, Shibuya A, et al: Radiofrequency ablation of hepatocellular carcinoma: correlation between loca tumor progression after ablation and ablative margin. AJR 188: 480-488, 2007.

7. Liu CH, Arellano RS, Uppot RN, Samir AE, Gervais DA and Mueller PR: Radiofrequency ablation of hepatic tumours: effect of post-ablation margin on local tumour progression. Eur Radiol 20: 879-885, 2009.

8. Denys AL, De Baere T, Kuoch V, et al: Radio-frequency tissue ablation of the liver: in vivo and ex vivo experiments with four different systems. Eur Radiol 13: 2346-2352, 2003.

9. Mendiratta-Lala M, Brook OR, Midkiff BD, et al: Quality initiatives: strategies for anticipating and reducing complications and treatment failures in hepatic radiofrequency ablation. Radiographics 30: 1107-1122, 2010.

10. Kobayashi M, Ikeda K, Someya T, et al: Stepwise hook extension technique for radiofrequency ablation therapy of hepatocellular carcinoma. Oncology 63: 139-144, 2002.

11. Choi D, Kim SK, Lim HK, et al: Overlapping ablation using a coaxial radiofrequency electrode and multiple cannulae system: experimental study in ex-vivo bovine liver. Korean J Radiol 4: 117-123, 2003

12. Goldberg SN, Gazelle GS, Dawson SL, Rittman WJ, Mueller PR and Rosenthal DI: Tissue ablation with radiofrequency: effect of probe size, gauge, duration, and temparature on lesion volume. Acad Radiol 2: 399-404, 1995.

13. Solazzo SA, Ahmed M, Liu Z, Hines-Peralta AU and Goldberg SN: High-power generator for radiofrequency ablation: larger electrodes and pulsing algorithms in bovine ex vivo and porcine in vivo settings. Radiology 242: 743-750, 2007.

14. Appelbaum L, Sosna J, Pearson R, et al: Algorithm optimization for multitined radiofrequency ablation: comparative study in ex vivo and in vivo bovine liver. Radiology 254: 430-440, 2010.
15. Berber E, Herceg NL, Casto KJ and Siperstein AE: Laparoscopic radiofrequency ablation of hepatic tumors. Surg Endosc 8: 390-396, 2004.

16. Kotoh K, Nakamuta M, Morizono S, et al: A multi-step, incremental expansion method for radio frequency ablation: potimization of the procedure to prevent increases in intra-tumor pressure and to reduce the ablation time. Liver Int 25: 542-547, 2005.

17. Patterson EJ, Scudamore $\mathrm{CH}$, Owen DA, Nagy AG and Buczkowski AK: Radiofrequency ablation of porcine liver in vivo: effects of blood flow and treatment time on lesion size. Ann Surg 227: 559-565, 1998.

18. Goldberg SN, Hahn PF, Halpern EF, Fogle RM and Gazelle GS Radio-frequency tissue ablation: effect of pharmacologic modulation of blood flow on coagulation diameter. Radiology 209: 761-767, 1998.

19. Lu DS, Raman SS, Vodopich DJ, Wang M, Sayre J and Lassman C: Effect of vessel size on creation of hepatic radiofrequency lesions in pigs: assessment of the 'heat sink' effect. Am J Roentgenol 178: 47-51, 2002.

20. Rossi S, Garbagnati F, Lencioni R, et al: Percutaneous radiofrequency thermal ablation of nonresectable hepatocellular carcinoma after occlusion of tumor blood supply. Radiology 217 : $119-126,2000$

21. Yamakado K, Nakatsuka A, Ohmori S, et al: Radiofrequency ablation combined with chemoembolization in hepatocellular carcinoma: treatment response based on tumor size and morphology. J Vasc Interv Radiol 13: 1225-1232, 2002.

22. Veltri A, Moretto P, Doriguzzi A, Pagano E, Carrara G and Gandini G: Radiofrequency thermal ablation (RFA) after transarterial chemoembolization (TACE) as a combined therapy for unresectable non-early hepatocellular carcinoma (HCC). Eur Radiol 16: 661-669, 2006. 